\title{
Conditioned two-dimensional simple random walk: Green's function and harmonic measure
}

\author{
Serguei Popov
}

November 14, 2019

Department of Statistics, Institute of Mathematics, Statistics and Scientific Computation, University of Campinas - UNICAMP, rua Sérgio Buarque de Holanda 651, 13083-859, Campinas SP, Brazil

e-mail: popov@ime.unicamp.br

\begin{abstract}
We study the Doob's $h$-transform of the two-dimensional simple random walk with respect to its potential kernel, which can be thought of as the twodimensional simple random walk conditioned on never hitting the origin. We derive an explicit formula for the Green's function of this random walk, and also prove a quantitative result on the speed of convergence of the (conditional) entrance measure to the harmonic measure (for the conditioned walk) on a finite set.
\end{abstract}

Keywords: transience, Doob's $h$-transform, entrance measure

AMS 2010 subject classifications: Primary 60J10. Secondary 60G50, $82 \mathrm{C} 41$.

\section{Introduction and main results}

In this paper, we derive some basic "potential-theoretic" results for the twodimensional simple random walk conditioned on never hitting the origin, namely

(i) we obtain an explicit expression for its Green's function;

(ii) we prove a quantitative result on the convergence of the conditional entrance measure to a finite set $A$ starting from a distant site to the harmonic measure $\widehat{\mathrm{hm}}_{A}$ of the conditioned walk. 
As usual, results on the Green's function and the control of the entrance measure are important tools in the study of properties of trajectories of the random walk. Regarding the above item (i), one can note that the expression for that Green's function was implicitly present in the paper [3]; however, here we give a more clean-and-straightforward derivation. Regarding item (ii), it is important to keep in mind that the harmonic measure $\widehat{\mathrm{hm}}_{A}$ with respect to the conditioned walk is generally different from the harmonic measure $\mathrm{hm}_{A}$ with respect to the simple random walk.

The two-dimensional simple random walk conditioned on never hitting the origin is the main ingredient in the construction of the two-dimensional random interlacements introduced in [3] and further studied in [2, 10, (by its turn, it is an extention of classical random intelacement model [1, 4, 11] to two dimensions). It then became evident that the conditioned walk (denoted by $\widehat{S}$ in this paper) is an interesting object on its own. We list its basic properties later in this section, but let us mention a few more advanced but surprizing ones [5, 9]:

- Although the conditioned walk itself is transient, it hits any fixed infinite subset of $\mathbb{Z}^{2}$ infinitely many times a.s. (i.e., any infinite set is recurrent for $\widehat{S}$ ).

- Fix a typical large set (e.g., a large disk or rectangle, a long segment, etc.). Then, the proportion of the sites of this set which are eventually visited by $\widehat{S}$ is close in distribution to the Uniform law on the unit interval.

- Let $M_{n}:=\min _{m \geq n}\left\|\widehat{S}_{m}\right\|$ be the future minimal distance to the origin for the conditioned walk (here and in the sequel $\|\cdot\|$ stands for the Euclidean norm). By transience, it is clear that $M_{n} \rightarrow \infty$ a.s., but this divergence occurs in a "highly irregular" way: for any $\delta>0$, it holds that $M_{n} \leq n^{\delta}$ and $M_{k} \geq k^{\frac{1}{2}-\delta}$, for infinitely many $n, k$.

- despite transience, two independent copies of the $\widehat{S}$-walks (starting from sites of the same parity) will meet infinitely many times a.s.; the same will happen for a pair of independent $\widehat{S}$-walk and simple random walk.

Also, the content of this paper is intended to be a part of the book [8], that the author is currently working on ${ }^{1}$

Now we give formal definitions. We will repeatedly use the big-O notation: $f(x)=O(g(x))$ as $x \rightarrow a$ means that $\limsup _{x \rightarrow a}\left|\frac{f(x)}{g(x)}\right|<\infty$, where $a \in \mathbb{R} \cup\{\infty\}$.

\footnotetext{
${ }^{1}$ comments on $[8]$ will be, of course, very welcome
} 
In the following, we denote by $\left(S_{n}, n \geq 0\right)$ the simple random walk in $\mathbb{Z}^{2}$. Let us define its potential kernel $a(\cdot)$ by

$$
a(x)=\sum_{k=0}^{\infty}\left(\mathbb{P}_{0}\left[S_{k}=0\right]-\mathbb{P}_{x}\left[S_{k}=0\right]\right), \quad x \in \mathbb{Z}^{2}
$$

By definition, it holds that $a(0)=0$, and one can show that the above series converges and that the resulting value is strictly positive for all $x \neq 0$ (here and in the sequel we refer to Section 4.4 of [6]). Also, the function $a$ is harmonic outside the origin, i.e.,

$$
a(x)=\frac{1}{4} \sum_{y \sim x} a(y) \quad \text { for all } x \neq 0 .
$$

It is possible to prove that, as $x \rightarrow \infty$,

$$
a(x)=\frac{2}{\pi} \ln \|x\|+\gamma^{\prime}+O\left(\|x\|^{-2}\right),
$$

where, $\gamma^{\prime}=\pi^{-1}(2 \gamma+\ln 8)$ with $\gamma=0.5772156 \ldots$ the Euler-Mascheroni constant, cf. Theorem 4.4.4 of [6].

Let $\tau$ and $\tau^{+}$be the entrance and the hitting times for the simple random walk:

$$
\tau_{A}=\min \left\{n \geq 0: S_{n} \in A\right\},
$$

and

$$
\tau_{A}^{+}=\min \left\{n \geq 1: S_{n} \in A\right\},
$$

and we let $\tau_{x}:=\tau_{\{x\}}, \tau_{x}^{+}:=\tau_{\{x\}}^{+}$. We also use the notations $\widehat{\tau}_{A}, \widehat{\tau}_{A}^{+}, \widehat{\tau}_{x}, \widehat{\tau}_{x}^{+}$when $S$ is substituted by $\widehat{S}$ in the above definitions. Observe that the harmonicity of $a$ outside the origin immediately implies that the process $a\left(S_{k \wedge \tau_{0}}\right)$ is a martingale. We will repeatedly use this fact in the sequel. What we will also repeatedly use, is that, due to (3),

$$
a(x+y)-a(x)=O\left(\frac{\|y\|}{\|x\|}\right)
$$

for all $x, y \in \mathbb{Z}^{2}$ such that (say) $\|x\|>2\|y\|$.

With some (slight) abuse of notation, we also consider the function

$$
a(r)=\frac{2}{\pi} \ln r+\gamma^{\prime}
$$


of a real argument $r \geq 1$. Note that, in general, $a(x)$ need not be equal to $a(\|x\|)$, although they are of course quite close for large $x$. The advantage of using this notation is e.g. that, due to (3) and (4), we may write (for fixed $x$ or at least $x$ such that $2\|x\| \leq r)$

$$
\sum_{y \in \partial \mathrm{B}(x, r)} \nu(y) a(y)=a(r)+O\left(\frac{\|x\| \vee 1}{r}\right) \quad \text { as } r \rightarrow \infty
$$

for any probability measure $\nu$ on $\partial \mathrm{B}(x, r)$.

Now we define the main object of study in this paper, the Doob's $h$-transform of the two-dimensional simple random walk with respect to the potential kernel $a$, which can be informally seen as "the simple random walk conditioned on never hitting the origin". It is a Markov chain $\left(\widehat{S}_{n}, n \geq 0\right)$ on $\mathbb{Z}^{2} \backslash\{0\}$, and with transition probability matrix

$$
\widehat{P}(x, y)= \begin{cases}\frac{a(y)}{4 a(x)}, & \text { if } x \sim y, x \neq 0 \\ 0, & \text { otherwise }\end{cases}
$$

(note that (2) implies that the above is a stochastic matrix indeed).

Following [3], we summarize the basic properties of the random walk $\widehat{S}$ :

(i) The walk $\widehat{S}$ is reversible, with the reversible measure $\mu(x)=a^{2}(x)$.

(ii) In fact, it can be represented as a random walk on the two-dimensional lattice with the set of conductances $\left(a(x) a(y), x, y \in \mathbb{Z}^{2}, x \sim y\right)$.

(iii) The process $1 / a\left(\widehat{S}_{n \wedge \widehat{\tau}_{\mathcal{N}}}\right)$ is a martingale, where $\mathcal{N}$ is the set of the four neighbours of the origin.

(iv) As a consequence (see e.g. Theorem 2.5.8 of [7]), the walk $\widehat{S}$ is transient.

(v) Moreover, for all $x \neq 0$

$$
\mathbb{P}_{x}\left[\widehat{\tau}_{x}^{+}<\infty\right]=1-\frac{1}{2 a(x)}
$$

and for all $x \neq y, x, y \neq 0$

$$
\mathbb{P}_{x}\left[\widehat{\tau}_{y}<\infty\right]=\mathbb{P}_{x}\left[\widehat{\tau}_{y}^{+}<\infty\right]=\frac{a(x)+a(y)-a(x-y)}{2 a(x)} .
$$


Next, we define the Green's function of the conditioned walk $\widehat{S}$ in the usual way: for $x, y \in \mathbb{Z}^{2} \backslash\{0\}$

$$
\widehat{G}(x, y)=\mathbb{E}_{x} \sum_{k=0}^{\infty} \mathbf{1}\left\{\widehat{S}_{k}=y\right\}
$$

i.e., $\widehat{G}(x, y)$ is the mean number of visits to $y$ starting from $x$ (counting the possible "visit" at time 0$)$. We are able to calculate this function in terms of the potential kernel $a$ :

Theorem 1.1. For all $x, y \in \mathbb{Z}^{2} \backslash\{0\}$ it holds that

$$
\widehat{G}(x, y)=\frac{a(y)}{a(x)}(a(x)+a(y)-a(x-y)) .
$$

It is easy to observe that (10) actually follows from (7)-(8) (which appear already in [3]). Indeed, since the total number of visits to $y$ has Geometric distribution with success parameter $\mathbb{P}_{y}\left[\widehat{\tau}_{y}^{+}=\infty\right]$ under $\mathbb{P}_{y}$, we have

$$
\widehat{G}(x, y)=\mathbb{P}_{x}\left[\widehat{\tau}_{y}<\infty\right] \times \frac{1}{\mathbb{P}_{y}\left[\widehat{\tau}_{y}^{+}=\infty\right]},
$$

which indeed leads to (10). However, the proof of (7)-(8) in [3] is somewhat involved and not very intuitive. Here, we take the "classical" route of first obtaining the expression for the Green's function; then, it is straightforward to derive (7)-(8) from it in the usual way.

Next, we define the capacity and the harmonic measure for the conditioned walk. As in [3] (formulas $(13)-(14)$ ), the capacity $\widehat{\operatorname{cap}}(\cdot)$ with respect to the conditioned walk is defined by

$$
\widehat{\operatorname{cap}}(A)=\sum_{y \in A} a^{2}(y) \widehat{\operatorname{Es}}_{A}(y)
$$

where

$$
\widehat{\mathrm{Es}}_{A}(x)=\mathbb{P}_{x}\left[\widehat{\tau}_{A}^{+}=\infty\right] \mathbf{1}\{x \in A\}
$$

is the escape probability from $x \in A$ (again, with respect to the conditioned walk). Being $\operatorname{cap}(\cdot)$ the capacity for the two-dimensional simple random walk (see Section 6.6 of [6]), it holds that

$$
\widehat{\operatorname{cap}}(A)=\operatorname{cap}(A \cup\{0\})
$$


for all $A \subset \mathbb{Z}^{2} \backslash\{0\}$, see Proposition 2.2 of [3]. Then, we also have the usual (as e.g. in Proposition 4.6.4 of [6]) transient-case relation

$$
\mathbb{P}_{x}\left[\widehat{\tau}_{A}<\infty\right]=\sum_{y \in A} \widehat{G}(x, y) \widehat{\operatorname{Es}}_{A}(y)=\sum_{y \in A} \frac{\widehat{G}(x, y)}{a^{2}(y)} \times a^{2}(y) \widehat{\operatorname{Es}}_{A}(y) .
$$

Now, let us define the harmonic measure with respect to the conditioned walk in the following way:

$$
\widehat{\operatorname{hm}}_{A}(y)=\frac{a^{2}(y) \widehat{\operatorname{Es}}_{A}(y)}{\widehat{\operatorname{cap}}(A)} .
$$

(One can also note the following fact about the relation of $\widehat{\mathrm{hm}}_{A}$ to the harmonic measure $\mathrm{hm}_{A}$ with respect to the simple random walk: $\widehat{\mathrm{hm}}_{A}$ is $\mathrm{hm}_{A}$ biased by $a$ by (13) and (15) of [3]; however, we will not need this fact in this paper.) For $x \in \mathbb{Z}^{2}$ and $A \subset \mathbb{Z}^{2}$ let us define $\operatorname{dist}(x, A)=\inf _{y \in A}\|x-y\| ; \operatorname{also}$, denote $\operatorname{diam}(A)=$ $\sup _{x, y \in A}\|x-y\|$. We then argue that $\widehat{\mathrm{hm}}_{A}$ is indeed "the conditional entrance measure from infinity", and, moreover, we obtain a quantitative result for the difference between the entrance measure of the conditioned walk to a finite set and the harmonic measure $\widehat{\mathrm{hm}}_{A}$ on this set:

Theorem 1.2. Assume that $A \subset \mathbb{Z}^{2} \backslash\{0\}$ is finite and $x \notin A$ is such that $\operatorname{dist}(x, A) \geq 12(\operatorname{diam}(A)+1)$. For all $y \in A$, we have

$$
\mathbb{P}_{x}\left[\widehat{S}_{\widehat{\tau}_{A}}=y \mid \widehat{\tau}_{A}<\infty\right]=\widehat{\operatorname{hm}}_{A}(y)\left(1+O\left(\frac{\operatorname{diam}(A)}{\operatorname{dist}(x, A)}\right)\right)
$$

Let us also mention that the term $O\left(\frac{\operatorname{diam}(A)}{\operatorname{dist}(x, A)}\right)$ is the same that one obtains in the corresponding result for the simple random walk, see Theorem 3.17 of [8].

\section{Some auxiliary definitions and results}

We use the following notations for $A \subset \mathbb{Z}^{2}: A^{c}=\mathbb{Z}^{2} \backslash A$ is the complement of $A$, $\partial A=\left\{x \in A\right.$ : there exist $y \in A^{c}$ such that $\left.x \sim y\right\}$ is the boundary of $A$, and $\partial_{e} A=\partial A^{c}$ is the external boundary of $A$; also, $\mathrm{B}(x, r)=\{y:\|y-x\| \leq r\}$ is the ball (disk) in $\mathbb{Z}^{2}$ and $\mathrm{B}(r)$ stands for $\mathrm{B}(0, r)$. Let us recall a few facts about hitting probabilities of (conditioned or not) simple random walks, and about the relationship between $S$ and $\widehat{S}$. (In the three following results, the error term $O(\cdot)$ is uniform with respect to the starting point $x$.) 
Lemma 2.1. Let $y \in \mathbb{Z}^{2}$ and $r \geq\|y\|$ (so that $0 \in \mathrm{B}(y, r)$ ). Assume that $x \in$ $\mathrm{B}(y, r)$ and $x \neq 0$. Then

$$
\mathbb{P}_{x}\left[\tau_{\partial \mathrm{B}(y, r)}<\tau_{0}^{+}\right]=\frac{a(x)}{a(r)+O\left(\frac{\|y\|+1}{r}\right)},
$$

as $r \rightarrow \infty$. The above also holds with $\tau_{\partial_{e} \mathrm{~B}(y, r)}$ on the place of $\tau_{\partial \mathrm{B}(y, r)}$.

Proof. This is (part of) Lemma 3.1 of [3].

Lemma 2.2. Assume $r \geq 1$ and $\|x\| \geq r+1$. We have

$$
\mathbb{P}_{x}\left[\widehat{\tau}_{\mathrm{B}(r)}=\infty\right]=1-\frac{a(r)+O\left(r^{-1}\right)}{a(x)} .
$$

Proof. This is Lemma 3.4 of [3].

For $D \subset \mathbb{Z}^{2}$, let $\Gamma_{D}^{(x)}$ be the set of all nearest-neighbour finite trajectories that start at $x \in D \backslash\{0\}$ and end when entering $\partial D$ for the first time; denote also $\Gamma_{y, R}^{(x)}=\Gamma_{\mathrm{B}(y, R)}^{(x)}$. For $\mathcal{H} \subset \Gamma_{D}^{(x)}$ write $S \in \mathcal{H}$ (respectively, $\widehat{S} \in \mathcal{H}$ ) if there exists $k$ such that $\left(S_{0}, \ldots, S_{k}\right) \in \mathcal{H}$ (respectively, $\left.\left(\widehat{S}_{0}, \ldots, \widehat{S}_{k}\right) \in \mathcal{H}\right)$. In the next result we show that $\mathbb{P}_{x}\left[S \in \cdot \mid \tau_{0}>\tau_{\partial \mathrm{B}(R)}\right]$ and $\mathbb{P}_{x}[\widehat{S} \in \cdot]$ are almost indistinguishable on $\Gamma_{0, R}^{(x)}$ (that is, the conditional law of $S$ almost coincides with the unconditional law of $\widehat{S}$ ), which justifies the intuition that $\widehat{S}$ is $S$ conditioned on never hitting the origin.

Lemma 2.3. Let $x \in \mathrm{B}(R) \backslash\{0\}$, and assume $\mathcal{H} \subset \Gamma_{0, R}^{(x)}$. We have

$$
\mathbb{P}_{x}\left[S \in \mathcal{H} \mid \tau_{0}>\tau_{\partial \mathrm{B}(R)}\right]=\mathbb{P}_{x}[\widehat{S} \in \mathcal{H}]\left(1+O\left((R \ln R)^{-1}\right)\right) .
$$

Proof. This is Lemma 3.3(i) of [3].

Now, we work with the Green's function of the conditioned walk. Observe that, by 10$)$, we have

$$
\frac{\widehat{G}(x, y)}{a^{2}(y)}=\frac{\widehat{G}(y, x)}{a^{2}(x)}=\frac{a(x)+a(y)-a(x-y)}{a(x) a(y)},
$$

and so it is natural to introduce new notation $\hat{g}(x, y)=\frac{\widehat{G}(x, y)}{a^{2}(y)}=\hat{g}(y, x)$ for the "symmetrized" conditional Green's function. 
At this point, let us recall that the function $1 / a(\cdot)$ is harmonid ${ }^{2}$ on $\mathbb{Z}^{2} \backslash(\mathcal{N} \cup\{0\})$, and observe that the Green's function $\widehat{G}(\cdot, y)$ is harmonic on $\mathbb{Z}^{2} \backslash\{0, y\}$ (this is an immediate consequence of the total expectation formula). It turns out that this "small" difference will be quite important: indeed, the latter fact will be operational in some places below, for applying the Optional Stopping Theorem in some particular settings. For future reference, we formulate the above fact in the equivalent form:

Proposition 2.4. For any $y \in \mathbb{Z}^{2} \backslash\{0\}$ it holds that the process $\left(\widehat{G}\left(\widehat{S}_{n \wedge \widehat{\tau}_{y}}, y\right), n \geq 0\right)$ is a martingale, as well as the process $\left(\hat{g}\left(\widehat{S}_{n \wedge \widehat{\tau}_{y}}, y\right), n \geq 0\right)$. Moreover, let us define

$$
\hat{\ell}(x, y)=1+\frac{a(y)-a(x-y)}{a(x)}=\frac{\widehat{G}(x, y)}{a(y)} .
$$

Then the process $\left(\hat{\ell}\left(\widehat{S}_{n \wedge \widehat{\tau}_{y}}, y\right), n \geq 0\right)$ is a martingale.

By the way, notice that

$$
\lim _{\|x\| \rightarrow \infty} \hat{\ell}(x, y)=0
$$

for any fixed $y$, so the last process is a "martingale vanishing at infinity", which makes it more convenient for applications via the Optional Stopping Theorem (so this is why we kept " $1+$ " in (20)).

Next, we need a few technical estimates on the function $\hat{g}$.

Lemma 2.5. There exist two positive constants $c_{1}, c_{2}$ such that, for all $x, y \in$ $\mathbb{Z}^{2} \backslash\{0\}$,

$$
\frac{c_{1}}{\ln (1+\|x\| \vee\|y\|)} \leq \hat{g}(x, y) \leq \frac{c_{2}}{\ln (1+\|x\| \vee\|y\|)}
$$

Proof. Assume without restricting generality that $\|x\| \geq\|y\|$ (recall that $\hat{g}(x, y)=$ $\hat{g}(y, x))$, and consider the following two cases.

Case 1: $\|y\|>\|x\|^{1 / 2}$. In this case $a(x)$ and $a(y)$ are of the same order, and, since $\|x-y\| \leq 2\|x\|$, due to (3), $a(x-y)-a(x)$ is bounded above by a positive constant; therefore, the expression $a(x)+a(y)-a(x-y)$ will be of order $\ln \|x\|$. This implies that $\hat{g}(x, y)$ will be of order $\frac{1}{\ln \|x\|}$ indeed.

Case 2: $\|y\| \leq\|x\|^{1 / 2}$. Here, (4) implies that $a(x)-a(x-y)=O\left(\frac{\|x\|^{1 / 2}}{\|x\|}\right)=$ $O\left(\|x\|^{-1 / 2}\right)$, so

$$
\hat{g}(x, y)=\frac{a(y)+O\left(\|x\|^{-1 / 2}\right)}{a(x) a(y)}=\frac{1}{a(x)}\left(1+O\left(\frac{1}{\|x\|^{1 / 2} \ln (1+\|y\|)}\right)\right),
$$

\footnotetext{
${ }^{2}$ with respect to the conditioned walk
} 
and this again implies 22 .

It will be important to have difference estimates for the function $\hat{g}$ as well:

Lemma 2.6. Assume that $x, y, z \in \mathbb{Z}^{2} \backslash\{0\}$ are distinct and such that $\|x-y\| \wedge$ $\|x-z\| \geq 5\|y-z\|$. Then

$$
|\hat{g}(x, y)-\hat{g}(x, z)| \leq O\left(\frac{\|y-z\|}{\|x-y\| \ln (1+\|x\| \vee\|y\| \vee\|z\|) \ln (1+\|y\| \vee\|z\|)}\right) .
$$

Proof. First, let us write

$$
\begin{aligned}
& \hat{g}(x, y)-\hat{g}(x, z) \\
& =\frac{a(x)+a(y)-a(x-y)}{a(x) a(y)}-\frac{a(x)+a(z)-a(x-z)}{a(x) a(z)} \\
& =\frac{a(x) a(z)-a(x-y) a(z)-a(x) a(y)+a(x-z) a(y)}{a(x) a(y) a(z)}
\end{aligned}
$$

(put $\pm a(x-z) a(z)$ to the numerator, then group accordingly)

$$
=\frac{a(x)(a(z)-a(y))-a(x-z)(a(z)-a(y))+a(z)(a(x-z)-a(x-y))}{a(x) a(y) a(z)} .
$$

Throughout this proof, let us assume without loss of generality that $\|y\| \geq\|z\|$. Since the walk $\widehat{S}$ is not spatially homogeneous (and, therefore, $\hat{g}$ is not translationally invariant), we need to take into account the relative positions of the three sites with respect to the origin. Specifically, we will consider the following three different cases (see Figure 1).

Case 1: $\|y\|^{1 / 2} \leq\|x\| \leq 2\|y\|$.

In this case, the first thing to note is that

$$
\|y-z\| \leq \frac{\|x-y\|}{5} \leq \frac{\|x\|+\|y\|}{5} \leq \frac{2\|y\|+\|y\|}{5}=\frac{3}{5}\|y\|,
$$

so $\|z\| \geq \frac{2}{5}\|y\|$, meaning that $\|y\|$ and $\|z\|$ must be of the same order; this then implies that $a(x), a(y), a(z)$ are all of the same order too. Then, we use (4) on the three parentheses in the numerator of (24), to obtain after some elementary calculations that the expression there is at most of order $\frac{\|y-z\|}{\|x-y\|} \ln \|y\|$, while the denominator is of order $\ln ^{3}\|y\|$. This proves (23) in case 1 .

Case 2: $\|x\|<\|y\|^{1 / 2}$. 

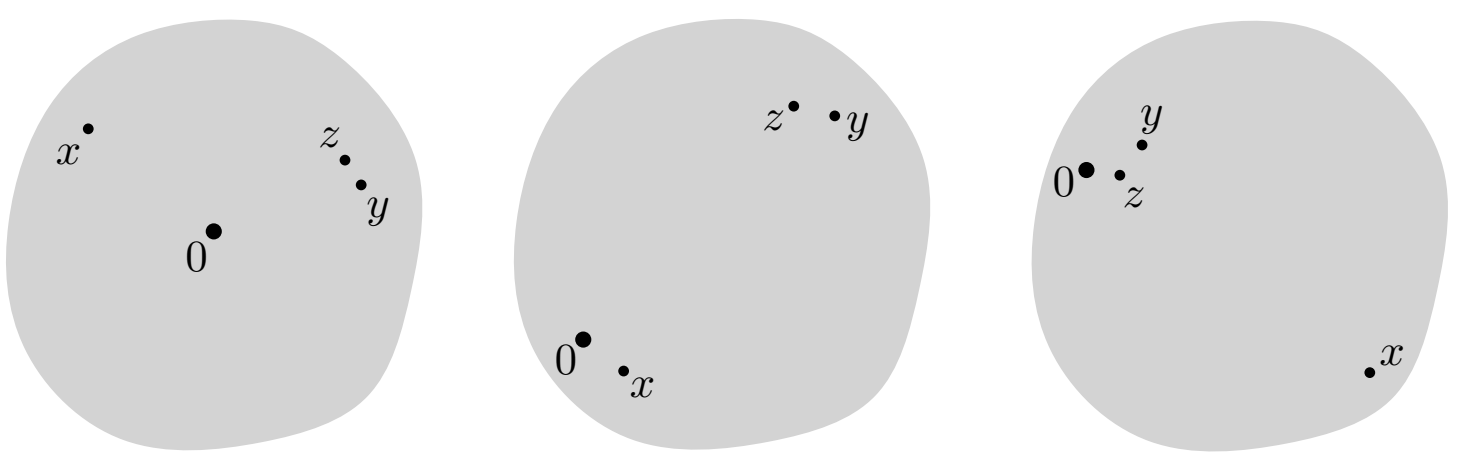

Figure 1: On the proof of Lemma 2.6, the three cases to consider (from left to right): (1) $\|x\|,\|y\|$ are of the same logarithmic order and $\|x\|$ is not much larger than $\|y\|,(2)\|x\|$ is much smaller than $\|y\|,(3)\|x\|$ is significantly larger than $\|y\|$.

Here, it is again easy to see that $\|y\|$ and $\|z\|$ must be of the same order. Now, we note that, by (4), $a(x-z)=a(z)+O\left(\frac{\|x\|}{\|y\|}\right)$, so, inserting this to (24) (and also using that $\left.a(y)-a(z)=O\left(\frac{\|y-z\|}{\|y\|}\right)\right)$, we find that it is equal to

$$
\begin{aligned}
& \frac{a(x)(a(z)-a(y))+a(z)(a(y)-a(z)+a(x-z)-a(x-y))+O\left(\frac{\|x\|}{\|y\|} \cdot \frac{\|y-z\|}{\|y\|}\right)}{a(x) a(y) a(z)} \\
& =\frac{a(z)-a(y)}{a(y) a(z)}+\frac{a(y)-a(z)+a(x-z)-a(x-y)}{a(x) a(y)}+O\left(\frac{\|x\| \cdot\|y-z\|}{\|y\|^{2} \ln ^{2}\|y\|}\right) .
\end{aligned}
$$

Now, by (4) the first term is $O\left(\frac{\|y-z\|}{\|y\| \ln ^{2}\|y\|}\right)$ (that is, exactly what we need, since $\|y\|$ and $\|y-x\|$ are of the same order), and the third term is clearly of smaller order. As for the second term, note that, by (3) and using the fact that \|\|$x-y\|\cdot\| z \|-$ $\|y\| \cdot\|x-z\| \mid \leq\|x\| \cdot\|y-z\|$ by the Ptolemy's inequality, we obtain

$$
\begin{aligned}
& a(y)-a(z)+a(x-z)-a(x-y) \\
& =\frac{2}{\pi} \ln \frac{\|y\| \cdot\|x-z\|}{\|x-y\| \cdot\|z\|}+O\left(\|y\|^{-2}\right) \\
& =\frac{2}{\pi} \ln \left(1-\frac{\|x-y\| \cdot\|z\|-\|y\| \cdot\|x-z\|}{\|x-y\| \cdot\|z\|}\right)+O\left(\|y\|^{-2}\right) \\
& =O\left(\frac{\|x\| \cdot\|y-z\|}{\|x-y\| \cdot\|z\|}+\|y\|^{-2}\right)=O\left(\frac{\|x\| \cdot\|y-z\|}{\|z\|^{2}}\right),
\end{aligned}
$$


so it is again of smaller order than the first term. This shows 23 in case 2.

Case 3: $\|x\|>2\|y\|$.

Notice that, in this case, $\|z\|$ need not be of the same order as $\|y\|$, it may happen to be significantly smaller. Here (by also grouping the first two terms in the numerator) we rewrite (24) as

$$
\frac{(a(x)-a(x-z))(a(z)-a(y))}{a(x) a(y) a(z)}+\frac{a(x-z)-a(x-y)}{a(x) a(y)} .
$$

By (4), the second term is $O\left(\frac{\|y-z\|}{\|x-y\| \ln (1+\|x\|) \ln (1+\|y\|)}\right)$ (that is, exactly what we need). Next, observe that (recall that we assumed that $\|y\| \geq\|z\|$ )

$$
\ln \|y\|-\ln \|z\|=\ln \frac{\|y\|}{\|z\|} \leq \frac{\|y\|}{\|z\|}-1=\frac{\|y\|-\|z\|}{\|z\|} \leq \frac{\|y-z\|}{\|z\|} .
$$

Therefore (also using (4) on the first factor), the numerator of the first term is $O\left(\frac{\|z\|}{\|x\|} \times \frac{\|y-z\|}{\|z\|}\right)=O\left(\frac{\|y-z\|}{\|x\|}\right)$, and so (since the denominator is not less than $\left.a(x) a(y)\right)$ the first term in (26) is at most of the same order as the second one. This concludes the proof of Lemma 2.6 .

Lemma 2.6 permits us to obtain the following useful expression for the probability of ever hitting $A$ from a distant site. Consider a finite $A \subset \mathbb{Z}^{2} \backslash\{0\}$ with $y_{0} \in A$ and note first that $\left\|y_{0}\right\|+\operatorname{diam}(A)$ will be of the same order regardless of the choice of $y_{0}$. Assume now that $\operatorname{dist}(x, A)>5 \operatorname{diam}(A)$; then, it also holds that $\|x\| \vee\|y\| \vee\|z\|$ is of the same order as $\|x\| \vee\left(\left\|y_{0}\right\|+\operatorname{diam}(A)\right)$ for any choice of $y, z \in A$. Indeed, trivially $\|x\| \vee\|y\| \vee\|z\| \leq\|x\| \vee\left(\left\|y_{0}\right\|+\operatorname{diam}(A)\right)$; on the other hand, $\|y\|+\operatorname{diam}(A)<\|y\|+\operatorname{dist}(x, A) \leq\|y\|+\|x-y\| \leq\|x\|+2\|y\| \leq$ $3(\|x\| \vee\|y\| \vee\|z\|)$. Then, note that $1+\|y\|+\|y-z\| \leq 1+2\|y\|+\|z\| \leq$ $3(1+\|y\| \vee\|z\|)<(1+\|y\| \vee\|z\|)^{3}$ (since the expression in parentheses is at least 2), so

$$
\frac{\|y-z\|}{\ln (1+\|y\| \vee\|z\|)} \leq 3 \frac{\|y-z\|}{\ln (1+\|y\|+\|y-z\|)} \leq 3 \frac{\operatorname{diam}(A)}{\ln (1+\|y\|+\operatorname{diam}(A))},
$$

where the second inequality is due to the fact that the function $f(x)=\frac{x}{\ln (a+x)}$ is increasing on $(0,+\infty)$ for any $a \geq 1$. So, recalling (11) and (14), we see that Lemma 2.6 gives us (in the case $\operatorname{dist}(x, A)>5 \operatorname{diam}(A))$ that

$\mathbb{P}_{x}\left[\widehat{\tau}_{A}<\infty\right]=\widehat{\operatorname{cap}}(A)\left(\hat{g}\left(x, y_{0}\right)+O\left(\frac{\operatorname{diam}(A)}{\operatorname{dist}(x, A) \ln \left(1+\|x\| \vee\left(\left\|y_{0}\right\|+\operatorname{diam}(A)\right)\right) \ln \left(1+\left(\left\|y_{0}\right\|+\operatorname{diam}(A)\right)\right)}\right)\right)$. 
The next technical fact that we need is that the conditioned walk can go out of an annulus with uniformly positive probability:

Lemma 2.7. Let $b, C$ be positive constants such that $1+b<C$, and assume that $r \geq 1, x_{0}, y_{0} \in \mathbb{Z}^{2} \backslash\{0\}$ are such that $x_{0} \in \mathrm{B}\left(y_{0}, C r\right)$ and $\left\|x_{0}-y_{0}\right\|>(1+b) r$. Then, there exists a constant $c^{\prime}>0$ (depending only on $b$ and $C$ ) such that

$$
\mathbb{P}_{x_{0}}\left[\widehat{\tau}_{\partial \mathrm{B}\left(y_{0}, C r\right)}<\widehat{\tau}_{\mathrm{B}\left(y_{0}, r\right)}\right] \geq c^{\prime} .
$$

Proof. Note that we can assume that $r$ is large enough, otherwise the uniform ellipticity of the conditioned walk will imply the claim. First of all, it is clear that the analogue of (28) holds for simple random walk, i.e., for all $r, x_{0}, y_{0}$ as above it holds that

$$
\mathbb{P}_{x_{0}}\left[\tau_{\partial \mathrm{B}\left(y_{0}, 2 C r\right)}<\tau_{\mathrm{B}\left(y_{0}, r\right)}\right] \geq c^{\prime \prime}
$$

for some $c^{\prime \prime}>0$ which depends only on $b$ and $C$. Now, the idea is to derive (28) from $(29)$. It holds that the weight $\widehat{P}_{\varrho}$ with respect to $\widehat{S}$ of a finite path $\varrho$ which starts at $x_{0}$ and does not pass through the origin equals $\frac{a\left(\varrho_{\text {end }}\right)}{a\left(x_{0}\right)} P_{\varrho}$, where $P_{\varrho}=\left(\frac{1}{4}\right)^{|\varrho|}$ (with $|\varrho|$ being the length of the path $\varrho$ and $\varrho$ end being the last site of it) is the weight of the same path with respect to the simple random walk. We can then write for any $R>0$ such that $x_{0} \in \mathrm{B}\left(y_{0}, R\right)$

$$
\begin{aligned}
& \mathbb{P}_{x_{0}}\left[\widehat{\tau}_{\partial \mathrm{B}\left(y_{0}, R\right)}<\widehat{\tau}_{\mathrm{B}\left(y_{0}, r\right)}\right] \\
& \geq \min _{z \in \partial \mathrm{B}\left(y_{0}, R\right)} \frac{a(z)}{a\left(x_{0}\right)} \times \mathbb{P}_{x_{0}}\left[\tau_{\partial \mathrm{B}\left(y_{0}, R\right)}<\tau_{\mathrm{B}\left(y_{0}, r\right)}, \tau_{\partial \mathrm{B}\left(y_{0}, R\right)}<\tau_{0}\right] \\
& \geq \min _{z \in \partial \mathrm{B}\left(y_{0}, R\right)} \frac{a(z)}{a\left(x_{0}\right)} \times\left(\mathbb{P}_{x_{0}}\left[\tau_{\partial \mathrm{B}\left(y_{0}, R\right)}<\tau_{\mathrm{B}\left(y_{0}, r\right)}\right]-\mathbb{P}_{x_{0}}\left[\tau_{0}<\tau_{\partial \mathrm{B}\left(y_{0}, R\right)}\right]\right) .
\end{aligned}
$$

Now, a key observation is that, for all large enough $r$, the property

$$
\frac{a(z)}{a\left(x_{0}\right)} \geq \frac{1}{2} \text { for all } z \in \partial \mathrm{B}\left(y_{0}, R\right)
$$

holds for either $R=C r$ or $R=2 C r$ (or both). Indeed, roughly speaking, for (31) to hold it would be enough that $\|z\|$ is of order $r+\left\|y_{0}\right\|$ for all $z \in \partial \mathrm{B}\left(y_{0}, R\right)$; this can be seen to be so in at least one of the above cases (look at the left side of Figure 2, if $\partial \mathrm{B}\left(y_{0}, C r\right)$ is "too close" to the origin, then $\partial \mathrm{B}\left(y_{0}, 2 C r\right)$ is not).

For definiteness, assume now that (31) holds for $R=2 C r$. By (30), we have then

$$
\mathbb{P}_{x_{0}}\left[\widehat{\tau}_{\partial \mathrm{B}\left(y_{0}, C r\right)}<\widehat{\tau}_{\mathrm{B}\left(y_{0}, r\right)}\right]
$$



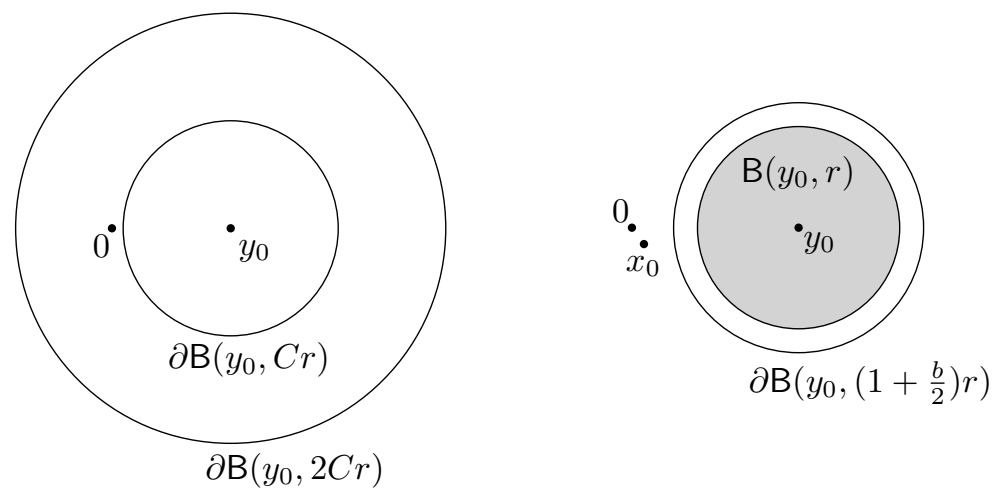

Figure 2: On the proof of Lemma 2.7

$$
\begin{aligned}
& \geq \mathbb{P}_{x_{0}}\left[\widehat{\tau}_{\partial \mathrm{B}\left(y_{0}, 2 C r\right)}<\widehat{\tau}_{\mathrm{B}\left(y_{0}, r\right)}\right] \\
& \geq \frac{1}{2}\left(\mathbb{P}_{x_{0}}\left[\tau_{\partial \mathrm{B}\left(y_{0}, 2 C r\right)}<\tau_{\mathrm{B}\left(y_{0}, r\right)}\right]-\mathbb{P}_{x_{0}}\left[\tau_{0}<\tau_{\partial \mathrm{B}\left(y_{0}, 2 C r\right)}\right]\right) \\
& \geq \frac{1}{2}\left(c^{\prime \prime}-\mathbb{P}_{x_{0}}\left[\tau_{0}<\tau_{\partial \mathrm{B}\left(y_{0}, 2 C r\right)}\right]\right) \\
& \geq \frac{c^{\prime \prime}}{4}
\end{aligned}
$$

provided that

$$
\mathbb{P}_{x_{0}}\left[\tau_{0}<\tau_{\partial \mathrm{B}\left(y_{0}, 2 C r\right)}\right] \leq \frac{c^{\prime \prime}}{2}
$$

Now, if $0 \notin \mathrm{B}\left(y_{0}, C r\right)$, then (32) trivially holds; so, let us assume that $0 \in$ $\mathrm{B}\left(y_{0}, C r\right)$. We then consider two cases: $\left\|x_{0}\right\| \geq \frac{b}{4} r$, and $\left\|x_{0}\right\|<\frac{b}{4} r$. In the first case, Lemma 2.1 implies that $\mathbb{P}_{x_{0}}\left[\tau_{0}<\tau_{\partial \mathrm{B}\left(y_{0}, 2 C r\right)}\right] \asymp \frac{1}{\ln r}$, so (32) holds for large enough $r$. In the second case, note first that

$$
\mathbb{P}_{x_{0}}\left[\widehat{\tau}_{\partial \mathrm{B}\left(y_{0}, C r\right)}<\widehat{\tau}_{\mathrm{B}\left(y_{0}, r\right)}\right] \geq \min _{z \in \partial \mathrm{B}\left(y_{0},\left(1+\frac{b}{2}\right) r\right)} \mathbb{P}_{z}\left[\widehat{\tau}_{\partial \mathrm{B}\left(y_{0}, C r\right)}<\widehat{\tau}_{\mathrm{B}\left(y_{0}, r\right)}\right]
$$

and, for all $z \in \partial \mathrm{B}\left(y_{0},\left(1+\frac{b}{2}\right) r\right)$, it holds that $\|z\| \geq \frac{b}{4} r$ (see Figure 2 on the right). We may then repeat the above reasoning with an arbitrary $z \in \partial \mathrm{B}\left(y_{0},\left(1+\frac{b}{2}\right) r\right)$ on the place of $x_{0}$ to finally obtain the claim.

Next technical fact we need is the following lower bound on the probability that the conditioned walk never hits a disk: 
Lemma 2.8. Fix $b>0$ and assume that $x_{0}, y_{0} \in \mathbb{Z}^{2} \backslash\{0\}$ and $r \geq 1$ are such that $\left\|x_{0}-y_{0}\right\| \geq(1+b) r$. Then there exists $c=c(b)$ such that for all $x_{0}, y_{0}, r$ as above it holds that

$$
\mathbb{P}_{x_{0}}\left[\widehat{\tau}_{\mathrm{B}\left(y_{0}, r\right)}=\infty\right] \geq \frac{c}{\ln \left(\left\|y_{0}\right\|+r\right)}
$$

Proof. We need to consider two cases: $\mathrm{B}\left(y_{0}, r\right)$ is (relatively to its size) close to/far from the origin. First, let us assume that $\left\|y_{0}\right\|<12 r$ (so that the disk is relatively close to the origin). In this case, we can assume additionally that $\left\|x_{0}-y_{0}\right\| \geq 51 r$ (indeed, Lemma 2.7 implies that, for any starting position $x_{0}$ such that $\left\|x_{0}-y_{0}\right\| \geq$ $(1+b) r$, with at least a constant probability the walk reaches $\partial \mathrm{B}\left(y_{0}, 51 r\right)$ before hitting $\left.\mathrm{B}\left(y_{0}, r\right)\right)$. Then, it holds that $\mathrm{B}\left(y_{0}, r\right) \subset \mathrm{B}(13 r)$, and $\mathrm{B}(26 r) \subset \mathrm{B}\left(y_{0}, 51 r\right)$. Now, Lemma 2.2 easily implies that, if $r \geq 1$ and $\|x\| \geq 2 r$

$$
\mathbb{P}_{x}\left[\widehat{\tau}_{\mathrm{B}(r)}=\infty\right] \geq \frac{c^{\prime}}{\ln r}
$$

(because (18) will work for large enough $\|x\|$, and one can use the uniform ellipticity of $\widehat{S}$ otherwise); this proves $(33)$ in the first case.

Now, suppose that $\left\|y_{0}\right\| \geq 12 r$ (that is, $r \leq \frac{1}{12}\left\|y_{0}\right\|$ ). Analogously to the previous case, Lemma 2.7 permits us to assume without loss of generality that $x_{0} \in \partial \mathrm{B}\left(y_{0}, 3 r\right)$. We now use the martingale (recall Proposition 2.4)

$$
\hat{\ell}\left(\widehat{S}_{n \wedge \widehat{\tau}_{y_{0}}}, y_{0}\right)=1+\frac{a\left(y_{0}\right)-a\left(\widehat{S}_{n \wedge \widehat{\tau}_{y_{0}}}-y_{0}\right)}{a\left(\widehat{S}_{n \wedge \widehat{\tau}_{y_{0}}}\right)} .
$$

The Optional Stopping Theorem implies that

$$
\begin{aligned}
\hat{\ell}\left(x_{0}, y_{0}\right) & =\sum_{z \in \partial \mathrm{B}\left(y_{0}, r\right)} \mathbb{P}_{x_{0}}\left[\widehat{\tau}_{\mathrm{B}\left(y_{0}, r\right)}<\infty, \widehat{S}_{\widehat{\tau}_{\mathrm{B}\left(y_{0}, r\right)}}=z\right] \hat{\ell}\left(z, y_{0}\right) \\
& \geq \mathbb{P}_{x_{0}}\left[\widehat{\tau}_{\mathrm{B}\left(y_{0}, r\right)}<\infty\right] \min _{z \in \partial \mathrm{B}\left(y_{0}, r\right)} \hat{\ell}\left(z, y_{0}\right),
\end{aligned}
$$

so

$$
\mathbb{P}_{x_{0}}\left[\widehat{\tau}_{\mathrm{B}\left(y_{0}, r\right)}<\infty\right] \leq \frac{\hat{\ell}\left(x_{0}, y_{0}\right)}{\min _{z \in \partial \mathrm{B}\left(y_{0}, r\right)} \hat{\ell}\left(z, y_{0}\right)} .
$$

Assume $z \in \partial \mathrm{B}\left(y_{0}, r\right)$ and write, using (3) and with $\gamma^{\prime \prime}:=\pi \gamma^{\prime} / 2$,

$$
\hat{\ell}\left(z, y_{0}\right)=\frac{a(z)+a\left(y_{0}\right)-a\left(y_{0}-z\right)}{a(z)}
$$




$$
\begin{aligned}
& =\frac{\ln \|z\|+\ln \left\|y_{0}\right\|-\ln r+\gamma^{\prime \prime}+O\left(\left\|y_{0}\right\|^{-2}+r^{-1}\right)}{\ln \|z\|+\gamma^{\prime \prime}+O\left(\|z\|^{-2}\right)} \\
& \geq \frac{\ln \left(\left\|y_{0}\right\|-r\right)+\ln \left\|y_{0}\right\|-\ln r+\gamma^{\prime \prime}+O\left(\left\|y_{0}\right\|^{-2}+r^{-1}\right)}{\ln \left(\left\|y_{0}\right\|+r\right)+\gamma^{\prime \prime}+O\left(\left\|y_{0}\right\|^{-2}+r^{-1}\right)} \\
& =\frac{2 \ln \left\|y_{0}\right\|+\ln \left(1-\frac{r}{\left\|y_{0}\right\|}\right)-\ln r+\gamma^{\prime \prime}+O\left(r^{-1}\right)}{\ln \left\|y_{0}\right\|+\ln \left(1+\frac{r}{\left\|y_{0}\right\|}\right)+\gamma^{\prime \prime}+O\left(r^{-1}\right)} \\
& :=\frac{T_{1}}{T_{2}}
\end{aligned}
$$

and, denoting $R:=\left\|x_{0}-y_{0}\right\|$ (so that $R=3 r+O(1)$ ),

$$
\begin{aligned}
\hat{\ell}\left(x_{0}, y_{0}\right) & =\frac{a\left(x_{0}\right)+a\left(y_{0}\right)-a\left(y_{0}-x_{0}\right)}{a\left(x_{0}\right)} \\
& =\frac{\ln \left\|x_{0}\right\|+\ln \left\|y_{0}\right\|-\ln R+\gamma^{\prime \prime}+O\left(\left\|y_{0}\right\|^{-2}+R^{-2}\right)}{\ln \left\|x_{0}\right\|+\gamma^{\prime \prime}+O\left(\left\|x_{0}\right\|^{-2}\right)} \\
& \leq \frac{\ln \left(\left\|y_{0}\right\|+R\right)+\ln \left\|y_{0}\right\|-\ln R+\gamma^{\prime \prime}+O\left(\left\|y_{0}\right\|^{-2}+R^{-2}\right)}{\ln \left(\left\|y_{0}\right\|-R\right)+\gamma^{\prime \prime}+O\left(\left\|y_{0}\right\|^{-2}+R^{-2}\right)} \\
& =\frac{2 \ln \left\|y_{0}\right\|+\ln \left(1+\frac{R}{\left\|y_{0}\right\|}\right)-\ln R+\gamma^{\prime \prime}+O\left(R^{-2}\right)}{\ln \left\|y_{0}\right\|+\ln \left(1-\frac{R}{\left\|y_{0}\right\|}\right)+\gamma^{\prime \prime}+O\left(R^{-2}\right)} \\
& :=\frac{T_{3}}{T_{4}} .
\end{aligned}
$$

Now, a straightforward calculation yields

$$
\frac{T_{2}}{T_{4}}=1+\frac{\ln \frac{1+r /\left\|y_{0}\right\|}{1-R /\left\|y_{0}\right\|}+O\left(r^{-1}\right)}{\ln \left\|y_{0}\right\|+\ln \left(1-\frac{R}{\left\|y_{0}\right\|}\right)+\gamma^{\prime \prime}+O\left(R^{-2}\right)},
$$

and

$$
\begin{aligned}
\frac{T_{3}}{T_{1}} & =1-\frac{\ln \left(\frac{R}{r} \cdot \frac{1-r /\left\|y_{0}\right\|}{1+R /\left\|y_{0}\right\|}\right)+O\left(r^{-1}\right)}{2 \ln \left\|y_{0}\right\|-\ln \frac{r}{1-r /\left\|y_{0}\right\|}+\gamma^{\prime \prime}+O\left(r^{-1}\right)} \\
& \leq 1-\frac{\ln \left(\frac{R}{r} \cdot \frac{1-r /\left\|y_{0}\right\|}{1+R /\left\|y_{0}\right\|}\right)+O\left(r^{-1}\right)}{2 \ln \left\|y_{0}\right\|+\gamma^{\prime \prime}} .
\end{aligned}
$$

Therefore, by (34) we have (after some elementary calculations)

$$
\mathbb{P}_{x_{0}}\left[\widehat{\tau}_{\mathrm{B}\left(y_{0}, r\right)}<\infty\right]
$$




$$
\begin{aligned}
& \leq \frac{T_{2}}{T_{4}} \times \frac{T_{3}}{T_{1}} \\
& \leq 1-\frac{\ln \left(\frac{R}{r} \cdot \frac{1-r /\left\|y_{0}\right\|}{1+R /\left\|y_{0}\right\|}\right)^{1 / 2}-\ln \frac{1+r /\left\|y_{0}\right\|}{1-R /\left\|y_{0}\right\|}+O\left((\ln r)^{-1}\right)}{\ln \left\|y_{0}\right\|\left(1+O\left(\frac{1}{\ln \left\|y_{0}\right\|}\right)\right)} .
\end{aligned}
$$

It remains only to observe that, if $r$ is large enough, the numerator in (37) is bounded from below by a positive constant: indeed, observe that $\frac{R}{r}$ is (asymptotically) $3, \frac{r}{\left\|y_{0}\right\|}$ and $\frac{R}{\left\|y_{0}\right\|}$ are at most $\frac{1}{12}$ and $\frac{1}{4}$ respectively, and

$$
\sqrt{3 \times \frac{1-\frac{1}{12}}{1+\frac{1}{4}}} \times \frac{1-\frac{1}{4}}{1+\frac{1}{12}}=\sqrt{\frac{891}{845}}>1 .
$$

This concludes the proof of Lemma 2.8 in the case when $r$ is large enough; the case of smaller values of $r$, though, can be easily reduced to the former one by using the uniform ellipticity of the $\widehat{S}$-walk.

\section{Proofs of the main results}

Proof of Theorem 1.1. First, we need a very simple general fact about hitting times of recurrent Markov chains:

Lemma 3.1. Let $\left(X_{n}\right)$ be a recurrent Markov chain on a state space $\Sigma$, and $x \in \Sigma$, $A, B \subset \Sigma$ are such that $A \cap B=\emptyset$ and $x \notin A \cup B$. Then

$$
\mathbb{P}_{x}\left[\tau_{A}<\tau_{B}\right]=\mathbb{P}_{x}\left[\tau_{A}<\tau_{B} \mid \tau_{x}^{+}>\tau_{A \cup B}\right]
$$

(that is, the events $\left\{\tau_{A}<\tau_{B}\right\}$ and $\left\{\tau_{x}^{+}>\tau_{A \cup B}\right\}$ are independent under $\mathbb{P}_{x}$ ).

Proof. This is almost evident, so we give only a sketch (see Figure 3): let $p:=$ $\mathbb{P}_{x}\left[\tau_{A}<\tau_{B} \mid \tau_{x}^{+}>\tau_{A \cup B}\right]$ be the value of the probability in the right-hand side of (38). At the moments when the walker visits $x$, it tosses a coin to decide if it will revisit it before coming to $A \cup B$, or not. When it decides to definitely leave $x$ for $A \cup B$, the probability of choosing $A$ is $p$, so it is $p$ overall.

We continue proving Theorem 1.1. Fix (a large) $R>0$, abbreviate $\Lambda_{R}=$ $\mathrm{B}(R) \backslash\{0\}$, and let us denote for $y \in \Lambda_{R}$

$$
N_{y, R}^{*}=\sum_{k=0}^{\tau_{\Lambda_{R}^{c}}} \mathbf{1}\left\{S_{k}=y\right\}
$$




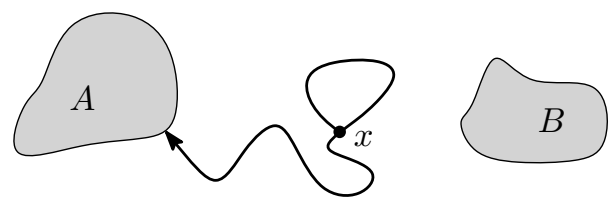

Figure 3: On the proof of Lemma 3.1

$$
\widehat{N}_{y, R}^{*}=\sum_{k=0}^{\widehat{\tau}_{\Lambda_{R}^{c}}} \mathbf{1}\left\{\widehat{S}_{k}=y\right\}
$$

to be the numbers of visits to $y$ before hitting 0 or $\partial_{e} \mathrm{~B}(R)$, for the simple random walk and the conditioned walk. Let us also denote $\widehat{G}_{R}(x, y)=\mathbb{E}_{x} \widehat{N}_{y, R}^{*}$. Now, let $x \in \Lambda_{R}$ and observe that, on one hand,

$$
\begin{aligned}
& \mathbb{P}_{x}\left[N_{y, R}^{*}=n, \tau_{\partial_{e} \mathrm{~B}(R)}<\tau_{0}\right] \\
& =\mathbb{P}_{x}\left[N_{y, R}^{*} \geq n\right] \mathbb{P}_{y}\left[\tau_{\partial_{e} \mathrm{~B}(R)}<\tau_{0}, \tau_{y}^{+}>\tau_{\Lambda_{R}^{c}}\right] \\
& =\mathbb{P}_{x}\left[N_{y, R}^{*} \geq n\right] \mathbb{P}_{y}\left[\tau_{y}^{+}>\tau_{\Lambda_{R}^{c}}\right] \mathbb{P}_{y}\left[\tau_{\partial_{e} \mathrm{~B}(R)}<\tau_{0} \mid \tau_{y}^{+}>\tau_{\Lambda_{R}^{c}}\right]
\end{aligned}
$$

(by Lemma 3.1

$$
\begin{aligned}
& =\mathbb{P}_{x}\left[N_{y, R}^{*} \geq n\right] \mathbb{P}_{y}\left[\tau_{y}^{+}>\tau_{\Lambda_{R}^{c}}\right] \mathbb{P}_{y}\left[\tau_{\partial_{e} \mathrm{~B}(R)}<\tau_{0}\right] \\
& =\mathbb{P}_{x}\left[N_{y, R}^{*}=n\right] \mathbb{P}_{y}\left[\tau_{\partial_{e} \mathrm{~B}(R)}<\tau_{0}\right]
\end{aligned}
$$

(by Lemma 2.1

$$
=\mathbb{P}_{x}\left[N_{y, R}^{*}=n\right] \frac{a(y)}{a(R)+O\left(R^{-1}\right)},
$$

and, on the other hand, the same expression can be also treated in the following way:

$$
\begin{aligned}
& \mathbb{P}_{x}\left[N_{y, R}^{*}=n, \tau_{\partial_{e} \mathrm{~B}(R)}<\tau_{0}\right] \\
& =\mathbb{P}_{x}\left[N_{y, R}^{*}=n \mid \tau_{\partial_{e} \mathrm{~B}(R)}<\tau_{0}\right] \mathbb{P}_{x}\left[\tau_{\partial_{e} \mathrm{~B}(R)}<\tau_{0}\right]
\end{aligned}
$$

(by Lemma 2.3

$$
=\mathbb{P}_{x}\left[\widehat{N}_{y, R}^{*}=n\right]\left(1+O\left((R \ln R)^{-1}\right)\right) \mathbb{P}_{x}\left[\tau_{\partial_{e} \mathrm{~B}(R)}<\tau_{0}\right]
$$


(again, by Lemma 2.1)

$$
=\mathbb{P}_{x}\left[\widehat{N}_{y, R}^{*}=n\right]\left(1+O\left((R \ln R)^{-1}\right)\right) \frac{a(x)}{a(R)+O\left(R^{-1}\right)} .
$$

Note also that $a(R)+O\left(R^{-1}\right)=a(R)\left(1+O\left((R \ln R)^{-1}\right)\right)$. So, since (39) and 40) must be equal, we have

$$
a(x) \mathbb{P}_{x}\left[\widehat{N}_{y, R}^{*}=n\right]=a(y) \mathbb{P}_{x}\left[N_{y, R}^{*}=n\right]\left(1+O\left((R \ln R)^{-1}\right)\right)
$$

multiplying by $n$ and summing in $n \geq 1$, we obtain

$$
a(x) \widehat{G}_{R}(x, y)=a(y) G_{\Lambda_{R}}(x, y)\left(1+O\left((R \ln R)^{-1}\right)\right) .
$$

Note that $\widehat{G}_{R}(x, y) \rightarrow \widehat{G}(x, y)$ as $R \rightarrow \infty$, due to the Monotone Convergence Theorem. Next, we are actually able to say something about $G_{\Lambda_{R}}(x, y)$ : by Proposition 4.6.2(b) of [6] it holds that

$$
G_{\Lambda_{R}}(x, y)=\mathbb{E}_{x} a\left(S_{\tau_{\Lambda_{R}^{c}}}-y\right)-a(x-y)
$$

(once again, by Lemma 2.1)

$$
\begin{aligned}
& =\frac{a(x)}{a(R)+O\left(R^{-1}\right)}\left(a(R)+O\left(\frac{\|y\|+1}{R}\right)\right) \\
& \quad+\left(1-\frac{a(x)}{a(R)+O\left(R^{-1}\right)}\right) a(y)-a(x-y) \\
& =a(x)+a(y)-a(x-y)+O\left(\frac{\|y\|+1}{R}+\frac{a(x) a(y)}{a(R)}\right) .
\end{aligned}
$$

Inserting this back to 41 and sending $R$ to infinity, we finally obtain 10 .

Proof of Theorem 1.2. Let us assume without restricting generality that $A$ contains at least two sites, so that $\operatorname{diam}(A) \geq 1$. For $x \notin A, y \in \partial A$, and $n \geq 1$, let us denote by $\Theta_{x y}^{(n)}$ the set of nearest-neighbour trajectories $\wp=\left(z_{0}, \ldots, z_{k}\right)$ such that

- $z_{0}=x, z_{k}=y$, and $z_{j} \notin A$ for all $j \leq k-1$, i.e., the trajectory ends on the first entrance to $A$, which takes place in $y$;

- $\sum_{j=0}^{k} \mathbf{1}\left\{z_{j}=x\right\}=n$, i.e., the trajectory visits $x$ exactly $n$ times (note that we $d o$ count $z_{0}=x$ as one visit); 
Let us also denote by

$$
\widehat{N}_{x}=\sum_{j=0}^{\infty} 1\left\{\widehat{S}_{j}=x\right\}
$$

the total number of visits to $x \notin A$, by

$$
\widehat{N}_{x}^{b}=\sum_{j=0}^{\widehat{\tau}_{A}^{+}-1} 1\left\{\widehat{S}_{j}=x\right\}
$$

the number of visits to $x$ before the first return to $A$, and by

$$
\widehat{N}_{x}^{\sharp}=\sum_{j=\widehat{\tau}_{A}^{+}}^{\infty} 1\left\{\widehat{S}_{j}=x\right\}
$$

the number of visits to $x$ after the first return to $A$ (naturally, setting $\widehat{N}_{x}^{\sharp}=0$ on $\left.\left\{\widehat{\tau}_{A}^{+}=\infty\right\}\right)$.

Recall that $P_{\wp}$ (respectively, $\widehat{P}_{\wp}$ ) is the weight of the trajectory $\wp$ with respect to the simple random walk (respectively, to the $\widehat{S}$-walk). First, it is clear that

$$
\mathbb{P}_{x}\left[\widehat{\tau}_{A}<\infty, \widehat{S}_{\widehat{\tau}_{A}}=y\right]=\sum_{n=1}^{\infty} \sum_{\wp \in \Theta_{x y}^{(n)}} \widehat{P}_{\wp}
$$

then, by the reversibility of the $\widehat{S}$-walk,

$$
\mathbb{P}_{y}\left[\widehat{N}_{x}^{b} \geq n\right] \frac{a^{2}(y)}{a^{2}(x)}=\sum_{\wp \in \Theta_{x y}^{(n)}} \widehat{P}_{\wp} .
$$

Now, we can write

$$
\begin{aligned}
& \mathbb{P}_{x}\left[\widehat{S}_{\widehat{\tau}_{A}}=y \mid \widehat{\tau}_{A}<\infty\right] \\
& =\frac{\mathbb{P}_{x}\left[\widehat{\tau}_{A}<\infty, \widehat{S}_{\widehat{\tau}_{A}}=y\right]}{\mathbb{P}_{x}\left[\widehat{\tau}_{A}<\infty\right]} \\
& =\frac{1}{\mathbb{P}_{x}\left[\widehat{\tau}_{A}<\infty\right]} \sum_{n=1}^{\infty} \frac{a^{2}(y)}{a^{2}(x)} \mathbb{P}_{y}\left[\widehat{N}_{x}^{b} \geq n\right] \\
& =\frac{a^{2}(y)}{a^{2}(x) \mathbb{P}_{x}\left[\widehat{\tau}_{A}<\infty\right]} \mathbb{E}_{y} \widehat{N}_{x}^{b}
\end{aligned}
$$




$$
\begin{aligned}
= & \frac{a^{2}(y)}{a^{2}(x) \mathbb{P}_{x}\left[\widehat{\tau}_{A}<\infty\right]}\left(\mathbb{E}_{y} \widehat{N}_{x}-\mathbb{E}_{y} \widehat{N}_{x}^{\sharp}\right) \\
= & \frac{a^{2}(y)}{\mathbb{P}_{x}\left[\widehat{\tau}_{A}<\infty\right]}\left(\frac{\widehat{G}(y, x)}{a^{2}(x)}-\sum_{z \in \partial A} \mathbb{P}_{y}\left[\widehat{\tau}_{A}^{+}<\infty, \widehat{S}_{\widehat{\tau}_{A}^{+}}=z\right] \frac{\widehat{G}(z, x)}{a^{2}(x)}\right) \\
= & \frac{a^{2}(y)}{\mathbb{P}_{x}\left[\widehat{\tau}_{A}<\infty\right]}\left(\hat{g}(y, x)-\sum_{z \in \partial A} \mathbb{P}_{y}\left[\widehat{\tau}_{A}^{+}<\infty, \widehat{S}_{\widehat{\tau}_{A}^{+}}=z\right] \hat{g}(z, x)\right) \\
= & \frac{a^{2}(y)}{\mathbb{P}_{x}\left[\widehat{\tau}_{A}<\infty\right]}\left(\hat{g}(y, x)\left(\widehat{\mathrm{Es}}_{A}(y)+\sum_{z \in \partial A} \mathbb{P}_{y}\left[\widehat{\tau}_{A}^{+}<\infty, \widehat{S}_{\widehat{\tau}_{A}^{+}}=z\right]\right)\right. \\
= & \frac{a^{2}(y) \hat{g}(y, x) \widehat{E s}}{\mathbb{P}_{x}\left[\widehat{\tau}_{A}<\infty\right]} \\
& \left.+\frac{a^{2}(y)}{\mathbb{P}_{x}\left[\widehat{\tau}_{A}<\infty\right]} \sum_{z \in \partial A} \mathbb{P}_{y}\left[\widehat{\tau}_{A}^{+}<\infty, \widehat{S}_{\widehat{\tau}_{A}^{+}}=z\right] \hat{g}(z, x)\right) \\
& \left.\widehat{\tau}_{A}^{+}<\infty, \widehat{S}_{\widehat{\tau}_{A}^{+}}=z\right](\hat{g}(y, x)-\hat{g}(z, x)) .
\end{aligned}
$$

By (27) and Lemma 2.5 it holds that

$$
\frac{a^{2}(y) \hat{g}(y, x) \widehat{\operatorname{Es}}_{A}(y)}{\mathbb{P}_{x}\left[\widehat{\tau}_{A}<\infty\right]}=\widehat{\operatorname{hm}}_{A}(y)\left(1+O\left(\frac{\operatorname{diam}(A)}{\operatorname{dist}(x, A)}\right)\right)
$$

Therefore, it only remains to show that the second term in $(42)$ is $O\left(\widehat{\operatorname{hm}}_{A}(y) \frac{\operatorname{diam}(A)}{\operatorname{dist}(x, A)}\right)$.

Now, we are going to use the Optional Stopping Theorem with the martingale $\widehat{M}_{n \wedge \widehat{\tau}_{x}}$ where

$$
\widehat{M}_{n}=\hat{g}(y, x)-\hat{g}\left(\widehat{S}_{n}, x\right)
$$

to estimate the second term in $(42)$. Recall that $y \in \partial A$, and let us define

$$
V=\partial \mathrm{B}(y, 2 \operatorname{diam}(A)),
$$

see Figure 4. Let $\tau=\widehat{\tau}_{A}^{+} \wedge \widehat{\tau}_{V}$. We have (note that $\tau<\widehat{\tau}_{x}$ )

$$
\begin{aligned}
0 & =\mathbb{E}_{y} \widehat{M}_{0} \\
& =\mathbb{E}_{y} \widehat{M}_{\tau} \\
& =\mathbb{E}_{y}\left(\widehat{M}_{\widehat{\tau}_{A}} \mathbf{1}\left\{\widehat{\tau}_{A}^{+}<\widehat{\tau}_{V}\right\}\right)+\mathbb{E}_{y}\left(\widehat{M}_{\widehat{\tau}_{V}} 1\left\{\widehat{\tau}_{V}<\widehat{\tau}_{A}^{+}\right\}\right)
\end{aligned}
$$




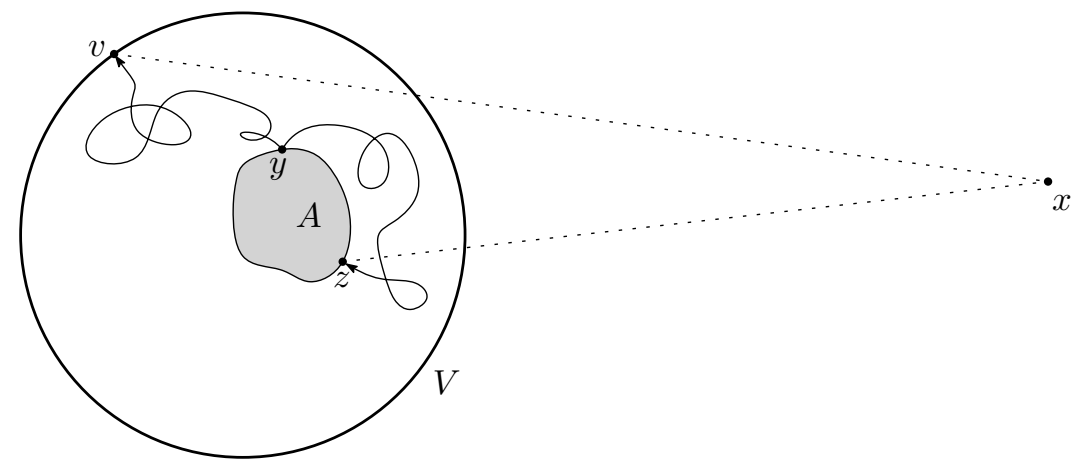

Figure 4: On the proof of Theorem 1.2 , the random walk starts at $y \in \partial A$ and ends either on the first re-entry to $A$ or entry to $V$.

$$
\begin{gathered}
\text { (since } \left.\mathbf{1}\left\{\widehat{\tau}_{A}^{+}<\infty\right\}=\mathbf{1}\left\{\widehat{\tau}_{A}^{+}<\widehat{\tau}_{V}\right\}+\mathbf{1}\left\{\widehat{\tau}_{V}<\widehat{\tau}_{A}^{+}<\infty\right\}\right) \\
=\mathbb{E}_{y}\left(\widehat{M}_{\widehat{\tau}_{A}^{+}} \mathbf{1}\left\{\widehat{\tau}_{A}^{+}<\infty\right\}\right)-\mathbb{E}_{y}\left(\widehat{M}_{\widehat{\tau}_{A}^{+}} \mathbf{1}\left\{\widehat{\tau}_{V}<\widehat{\tau}_{A}^{+}<\infty\right\}\right) \\
\quad+\mathbb{E}_{y}\left(\widehat{M}_{\widehat{\tau}_{V}} \mathbf{1}\left\{\widehat{\tau}_{V}<\widehat{\tau}_{A}^{+}\right\}\right) .
\end{gathered}
$$

Note that for any $z \in V \cup \partial A$ it holds that $\|y-z\| \leq 2 \operatorname{diam}(A)$ and $\|x-z\| \geq$ $10(\operatorname{diam}(A)+1)$, so in the following we will be able to apply Lemma 2.6. Since

$$
\mathbb{E}_{y}\left(\widehat{M}_{\widehat{\tau}_{A}^{+}} \mathbf{1}\left\{\widehat{\tau}_{A}^{+}<\infty\right\}\right)=\sum_{z \in \partial A} \mathbb{P}_{y}\left[\widehat{\tau}_{A}^{+}<\infty, \widehat{S}_{\widehat{\tau}_{A}^{+}}=z\right](\hat{g}(y, x)-\hat{g}(z, x)),
$$

we obtain that

$$
\begin{aligned}
& \sum_{z \in \partial A} \mathbb{P}_{y}\left[\widehat{\tau}_{A}^{+}<\infty, \widehat{S}_{\widehat{\tau}_{A}^{+}}=z\right](\hat{g}(y, x)-\hat{g}(z, x)) \\
& =\mathbb{E}_{y}\left(\widehat{M}_{\widehat{\tau}_{A}^{+}} \mathbf{1}\left\{\widehat{\tau}_{V}<\widehat{\tau}_{A}^{+}<\infty\right\}\right)-\mathbb{E}_{y}\left(\widehat{M}_{\widehat{\tau}_{V}} \mathbf{1}\left\{\widehat{\tau}_{V}<\widehat{\tau}_{A}^{+}\right\}\right) \\
& =\mathbb{P}_{y}\left[\widehat{\tau}_{V}<\widehat{\tau}_{A}^{+}\right]\left(\mathbb{E}_{y}\left(\widehat{M}_{\widehat{\tau}_{A}^{+}} \mathbf{1}\left\{\widehat{\tau}_{A}^{+}<\infty\right\} \mid \widehat{\tau}_{V}<\widehat{\tau}_{A}^{+}\right)-\mathbb{E}_{y}\left(\widehat{M}_{\widehat{\tau}_{V}} \mid \widehat{\tau}_{V}<\widehat{\tau}_{A}^{+}\right)\right)
\end{aligned}
$$

(by Lemma 2.6 and the derivation of 27]; recall that $\widehat{M}_{\tau}=\hat{g}(y, x)-\hat{g}(z, x)$ on $\left\{\widehat{S}_{\tau}=z\right\}$ )

$$
\leq \mathbb{P}_{y}\left[\widehat{\tau}_{V}<\widehat{\tau}_{A}^{+}\right] \times O\left(\frac{\operatorname{diam}(A)}{\operatorname{dist}(x, A) \ln (1+\|y\|+\operatorname{diam}(A)) \ln (1+\|x\| \vee(\|y\|+\operatorname{diam}(A)))}\right) .
$$

Next, we can write

$$
\widehat{\mathrm{Es}}_{A}(y)=\mathbb{P}_{y}\left[\widehat{\tau}_{A}^{+}=\infty\right]
$$




$$
=\sum_{v \in V} \mathbb{P}_{y}\left[\widehat{\tau}_{V}<\widehat{\tau}_{A}^{+}, \widehat{S}_{\widehat{\tau}_{V}}=v\right] \mathbb{P}_{v}\left[\widehat{\tau}_{A}=\infty\right]
$$

(by Lemma 2.8)

$$
\geq \frac{c}{\ln (\|y\|+\operatorname{diam}(A))} \mathbb{P}_{y}\left[\widehat{\tau}_{V}<\widehat{\tau}_{A}^{+}\right]
$$

which means that

$$
\mathbb{P}_{y}\left[\widehat{\tau}_{V}<\widehat{\tau}_{A}^{+}\right] \leq O\left(\widehat{\operatorname{Es}}_{A}(y) \ln (\|y\|+\operatorname{diam}(A))\right)
$$

Also, 43 implies that

$$
\frac{a^{2}(y)}{\mathbb{P}_{x}\left[\widehat{\tau}_{A}<\infty\right]}=O\left(\frac{\widehat{\mathrm{hm}}_{A}(y)}{\widehat{\operatorname{Es}}_{A}(y) \hat{g}(x, y)}\right)
$$

Since, by Lemma 2.5 .

$$
\frac{1}{\hat{g}(x, y)}=O(\ln (1+\|x\| \vee\|y\|)),
$$

it only remains to combine (44)-46) to see that the second term in 42 is indeed $O\left(\widehat{\operatorname{hm}}_{A}(y) \frac{\operatorname{diam}(A)}{\operatorname{dist}(x, A)}\right)$, thus concluding the proof of Theorem 1.2 .

\section{Acknowledgments}

This work was partially supported by CNPq (301605/2015-7). The author is grateful to the anonymous referee for many comments and suggestions on the first version of this paper.

\section{References}

[1] J. Černý, A. TeixeIRA (2012) From random walk trajectories to random interlacements. Ensaios Matemáticos [Mathematical Surveys] 23. Sociedade Brasileira de Matemática, Rio de Janeiro.

[2] F. Comets, S. Popov (2017) The vacant set of two-dimensional critical random interlacement is infinite. Ann. Probab. 45, 4752-4785. 
[3] F. Comets, S. Popov, M. Vachkovskaia (2016) Two-dimensional random interlacements and late points for random walks. Commun. Math. Phys. 343, 129-164.

[4] A. Drewitz, B. RÁth, A. Sapozhnikov (2014) An Introduction to Random Interlacements. Springer.

[5] N. Gantert, S. Popov, M. Vachkovskaia (2019) On the range of a two-dimensional conditioned simple random walk. Ann. Henri Lebesgue 2, 349-368.

[6] G. Lawler, V. Limic (2010) Random walk: a modern introduction. Cambridge Studies in Advanced Mathematics, 123. Cambridge University Press, Cambridge.

[7] M. Menshikov, S. Popov, A. Wade (2017) Non-homogeneous Random Walks: Lyapunov Function Methods for Near-Critical Stochastic Systems Cambridge University Press, Cambridge.

[8] S. Popov (2019) Simple Random Walk in Two Dimensions. Work in progress. Available at: www.ime.unicamp.br/ popov/2srw.pdf

[9] S. Popov, L.T. Rolla, D. Ungaretti (2019) Quantifying the transience of conditioned walks on the plane: encounters and speed of escape. Work in progress.

[10] P.-F. Rodriguez (2019) On pinned fields, interlacements, and random walk on $(\mathbb{Z} / N \mathbb{Z})^{2}$. Probab. Theory Relat. Fields 173 (3), 1265-1299.

[11] A.-S. Sznitman (2010) Vacant set of random interlacements and percolation. Ann. Math. (2), 171 (3), 2039-2087. 